\title{
Comparison between Thermography and Fluorescein Test in the Detection of Incompetent Perforating Veins
}

\section{Summary}

Incompetent perforating veins of the legs were located by dynamic thermography at 69 sites and by the fluorescein test at 31. All the sites were explored by multiple local incisions. Incompetence of the veins was determined by demonstrating retrograde blood flow through these veins when severed. Explorations of these sites showed that thermography detected $94 \%$ and gave false-positive results in $6 \%$, while the fluorescein test detected $16 \%$ and contributed $84 \%$ false-positive results. These findings show a highly significant difference $(P<0.0005)$ in favour of the thermographic technique. Detection of $16 \%$ of incompetent perforating veins by the fluorescein test is statistically insignificant. This inaccuracy is thought to be due to inadequate dermal penetration of the ultraviolet rays. A positive fluorescein test probably indicates the presence of incompetent perforating veins but has little anatomical relationship to their actual site.

\section{Introduction}

To locate accurately the incompetent perforating veins is vital for successful treatment of varicose veins of the lower limbs. Ligation (Linton, 1938; Cockett and Jones, 1953) and compression sclerotherapy (Fegan and Fitzgerald, 1963) are designed to ligate or sclerose these veins. Sites of the incompetent perforating veins may be located clinically and by special tests. By careful clinical examination between $51 \%$ (Beesley and Fegan, 1970) and 69\% (Townsend et al., 1967) of the incompetent perforating veins may be correctly located. Special methods of investigation include venography, dynamic thermography, and the fluorescein test.

Phlebography can demonstrate $92 \%$ (Townsend et al., 1967) and dynamic thermography can demonstrate $95 \%$ (Patil et al., 1970) of the incompetent perforating veins. Accuracy of the fluorescein test (Chilvers and Thomas, 1970) is stated to be $96 \%$. Effectiveness of phlebography is diminished by difficulty in locating the actual sites of these veins on the limbs from two-dimensional $x$-ray films. The high accuracy of location by dynamic thermography has often been discounted because of the formidable initial expense of procuring a modern infrared scanner. The fluorescein test is easy to perform and the least expensive. The simplicity and stated accuracy of $96 \%$ of the fluorescein test in theory makes it an attractive test for locating incompetent perforating veins. This study was undertaken to compare the accuracy of the fluorescein test with dynamic thermography in locating incompetent perforating veins of the lower limbs.

\section{Procedure}

\section{TECHNIQUE OF THERMOGRAPHIC EXAMINATION}

With the patient in the supine position on a couch, the leg to be examined is raised to an angle of $45^{\circ}$ by supporting the

\footnotetext{
Department of Measurement and Clinical Research, St. Martin's Hospital, Bath

B. ELEM, M.B., B.S., F.R.C.s., Clinical Research Registrar

B. A. SHÓREY, M.B., B.S., F.R.C.S., Surgical Registrar

K. LLOYD WILLIAMS, M.CHIR., F.R.c.s., Consultant Surgeon
}

heel on a stool placed on the foot end of the couch. The scanner camera is positioned so that the scanner screen displays the whole of the examining leg (from the ankle to the knee) with sharply demarcated borders. The superficial veins and any obvious varices are emptied by raising the leg vertically upwards for one minute. With the leg raised, tourniquets are applied just above the medial malleolus and below the knee and inflated to $80 \mathrm{~mm} \mathrm{Hg}$. The leg is then lowered to its original

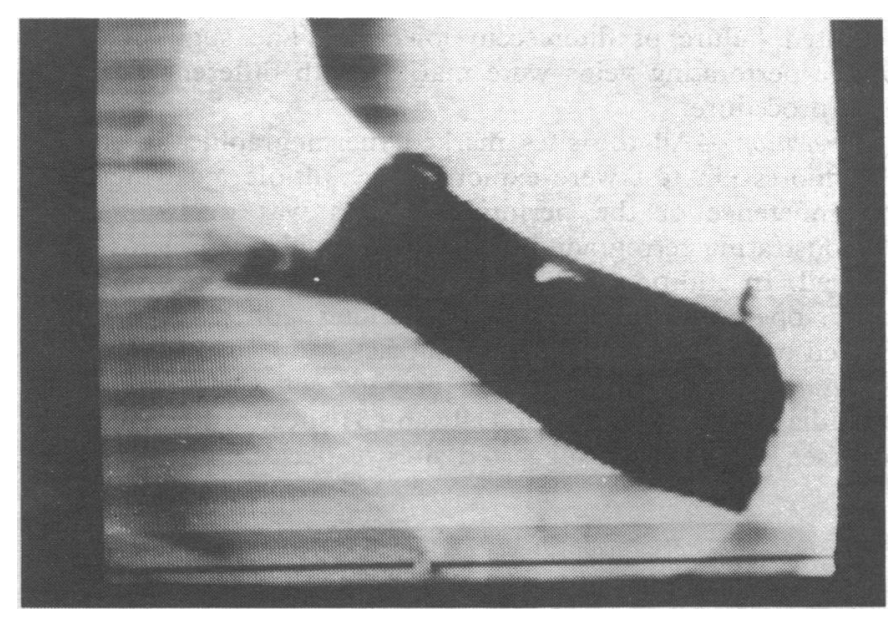

Thermogram showing a suggested site of an incompetent perforating vein in the leg (hot and cold areas are shown in white and dark shades vein in the le
respectively).

position. The part to be examined is cooled by gently rubbing the skin with ice cubes until it assumes a uniformly dark colour on the scanner screen. The patient then plantar flexes the foot for 60 seconds, and the appearance of hot (white) circular areas 0.5 to $2 \mathrm{~cm}$ in diameter (see Figure) during this time suggests the possible sites of incompetent perforating veins. The areas on the leg corresponding to the hot areas on the screen are marked with a felt pen containing indelible ink. Examination is repeated to cover the medial, lateral, and posterior aspects of the leg. Examination of both legs may be completed in 30 minutes. The inner aspect of the thigh may be examined similarly, from knee to groin. Tourniquets are applied at the thigh and knee, and active quadriceps exercise is substituted for plantar flexion of the foot.

This procedure can be repeated as often as required if the presence or absence of an incompetent perforator at any site is in question.

\section{TECHNIQUE OF FLUORESCEIN TEST}

The technique used was that suggested by Chilvers and Thomas (1970). The patient lies on a couch with the foot tilted down $10^{\circ}$. A $2 \cdot 5-\mathrm{cm}$ sphygmomanometer cuff is placed around the ankle and inflated to $80 \mathrm{~mm} \mathrm{Hg}$. A second sphygmomanometer cuff of normal width is placed above the knee. The leg is raised vertically and the patient plantar flexes the foot 10 times in order to empty the superficial varices and to pump venous blood out of the calf. The upper cuff is then inflated to $120 \mathrm{~mm} \mathrm{Hg}$ and the leg lowered. Into a vein, preferably on the most distal and lateral part of the foot, $5 \mathrm{ml}$ of $5 \%$ aqueous fluorescein is injected slowly (30 seconds). The room is blacked out and an ultraviolet light (Hanovia-Kromayer 
$1 \times$ SK220 with Wood's filter) is shone on the leg from a distance of $3 \mathrm{ft}(30 \mathrm{~cm})$. The patient plantar flexes his foot 10 times to facilitate drawing the dye into the deep venous system. The sites of incompetent perforating veins are shown by a small circle of green-yellow fluorescence $1-2 \mathrm{~cm}$ in diameter. The fluorescent areas may take between 30 seconds and two minutes to appear.

\section{Clinical Material and Methods}

Patients with clinical evidence of incompetent perforating veins were selected. No attempt was made clinically to locate the incompetent sites. Eighteen patients $(11$ female and 7 male) were included in the trial. Altogether 25 limbs were investigated. Fluorescein and thermographic examinations as described previously were carried out in all the limbs except one, in which thermography alone was performed owing to repeated failure of fluorescein injection. The suggested sites of the perforating veins were marked with different signs for each procedure.

Operation.-All the sites marked thermographically and by the fluorescein test were explored by multiple local incisions. Incompetence of the perforating veins was determined by demonstrating retrograde blood flow through these veins when severed. In addition to ligating incompetent veins Trendelenburg's operation and stripping of the long saphenous vein were carried out when considered appropriate.

Results.-Sixty-nine sites of incompetent perforating veins were diagnosed thermographically and 31 sites by the fluorescein test (see Table). Five suggested sites of perforating veins were

Suggested Sites of Incompetent Perforating Veins by Thermography and Fluorescein Test and Their Anatomical Location at Operation.

\begin{tabular}{|c|c|c|c|}
\hline Methods & $\begin{array}{l}\text { Total } \\
\text { Suggested } \\
\text { Sites }\end{array}$ & Found at Operation & $\begin{array}{l}\text { Not Found at } \\
\text { Operation }\end{array}$ \\
\hline $\begin{array}{l}\text { Thermography } \\
\text { Fluorescein test }\end{array}$ & $\begin{array}{l}69 \\
31\end{array}$ & $\begin{array}{r}65(94 \cdot 2 \%) \\
5(16 \cdot 12 \%)\end{array}$ & $\begin{array}{r}4\left(5 \cdot 799^{\prime}\right) \\
26\left(83.877_{0}^{\prime}\right)\end{array}$ \\
\hline
\end{tabular}

$P<0.0005$.

diagnosed by both thermography and fluorescein test. At exploration of the marked sites a total of 65 incompetent perforating veins was found. All of these veins were correctly located by thermography, giving a thermographic accuracy rate of $94 \%$. Out of 31 suggested sites diagnosed by fluorescein tests $5(16 \%)$ were found at operation. These five sites were also diagnosed by thermography. At operation $4(5 \%)$ of the sites suggested by thermography and $26(84 \%)$ by the fluorescein test did not have any incompetent perforating vein. Incomplete emptying of the visible varices was responsible for thermographic false-positive results. All the fluorescein false-positive sites showed at exploration a superficial venous plexus without any demonstrable incompetent perforating veins within 2 in $(5 \mathrm{~cm})$ of the fluorescein mark.

\section{Discussion}

\section{THERMOGRAPHY}

The location of incompetent perforating veins by thermography is based on two physiological factors: (1) the temperature in the deep veins is higher than that in the superficial system, and (2) during exercise retrograde flow of warm blood occurs through incompetent perforating veins (Lloyd Williams, 1964), giving rise to higher skin temperature.

With the use of line scans the total change during exercise in skin temperature overlying an incompetent perforating vein was found to range from 1.5 to $6^{\circ} \mathrm{C}$ at the end of 60 seconds
(Patil and Lloyd Williams, 1971). The rewarming of a cold leg always begins at the sites of incompetent perforating veins and gradually spreads to the adjacent areas.

In our study we confirm the previously reported thermographic accuracy of $94 \%$ (Patil et al., 1970).

\section{FLUORESCEIN}

When radiation falls on the surface of a fluorescent object it is absorbed and re-emitted as rays of longer wavelength. Thus ultraviolet rays falling on a fluorescent solution are absorbed and converted into visible light of longer wavelength. Application of this principle forms the basis of the fluorescein test. However, aqueous fluorescein injected into a segment of a visible superficial vein isolated between tourniquets in a thin person fails to fluoresce. This is due to the subdermal location of the vein containing fluorescein and the fact that ultraviolet radiation is all absorbed between the stratum corneum and the dermis. Hence the fluorescent circular areas noted in patients with incompetent perforating veins must be due to fluorescein in veins which lie wtthin the penetration depth of the ultraviolet radiation - that is, they must be the subepidermal venous plexus. It would be interesting to speculate how the fluorescein reaches the skin.

During muscular exercise high-pressure retrograde leakage of blood from the deep veins occurs into the superficial venous system through incompetent and dilated ankle perforators. This leads to a gradual dilatation of the subcutaneous and subepidermal venous and capillary plexuses (Dodd and Cockett, 1956; Arnoldi, 1966). Thus fluorescein solution injected into a dorsal pedal vein may reach the skin (subepidermal plexus) by retrograde flow through an incompetent perforating vein. However, it is inconceivable that fluorescein will bypass the maze of dilated venules and capillaries and will always reach only an area of skin $(1-2 \mathrm{~cm})$ corresponding to the actual site of an incompetent perforating vein. Thus a fluorescent site in the skin probably indicates the presence of an incompetent perforating vein in the limb but has little anatomical relationship to its actual site.

In some cases fluorescein solution may reach the skin by leakage of dye (under the ankle tourniquet) into the superficial system and spread in a retrograde manner within its superficial tributaries. This retrograde flow may be initiated by the raised pressure within the superficial system and is probably augmented by an above-knee tourniquet.

In our view the use of the fluorescein test as a method of actually locating an incompetent perforator is grossly inaccurate. Occasional operative findings of an incompetent perforating vein corresponding to a fluorescent site is a chance correlation rather than due to any sound pathophysiological reason.

We wish to thank the Department of Health and Social Security for the loan of the Rank Thermographic System and for the grant to carry out this work.

\section{References}

Arnoldi, C. C. (1966). Acta Chururgica Scandinavica, 132, 628.
Beesley, W. H., and Fegan, W. G. (1970). British fournal of Surgery, 57, 30. Beesley, W. H., and Fegan, W. G. (1970). British fournal of Surgery, 57, 30.

Dodd, H., and Cockett, F. B. (1956). The Pathology and Surgery of the Veins of the Lower Limbs. Edinburgh, Livingstone.
. B. (1956). The Pathology

Fegan, W. G., and Fitzgerald, D. E. (1963). Irish fournal of Medical Science, p. 439.

Linton, R. R. (1938). Annals of Surgery, 107, 582.

Lloyd Williams, K. (1964). Annals of the New York Academy of Sciences, 121,99

Patil, K. D., and Lloyd Williams, K. (1971). Surgery, Gynecology and Obstetrics, 132, 396

Patil, K. D., Williams, J. R., and Lloyd Williams, K. (1970). British Medical Fournal, 1, 195.

Townsend, J., Jones, H., and Williams, J. E. (1967). British Medical fournal, 3, 583 . 